\title{
ESTADO DO CONHECIMENTO SOBRE SOBRECARGA DE INFORMAÇÃO NA TOMADA DE DECISÃO EM ARTIGOS CIENTÍFICOS NO PERÍODO ENTRE 2007 E 2016
}

Icaro De Oliveira Vieira ${ }^{1}$

Edelvino Razzolini Filho ${ }^{1}$

${ }^{1}$ Universidade Federal do Paraná 


\section{ESTADO DO CONHECIMENTO SOBRE SOBRECARGA DE INFORMAÇÃO NA TOMADA DE DECISÃO EM ARTIGOS CIENTÍFICOS NO PERÍODO ENTRE 2007 E 2016}

Resumo: Este artigo busca descrever o estado do conhecimento sobre sobrecarga de informação na tomada de decisão em artigos científicos entre 2007 a 2016. Os dados analisados foram obtidos das bases de dados ScienceDirect e EBSCOHost. Foi utilizada a metodologia multicritério para a composição de um portfólio bibliográfico denominada Methodi Ordinatio desenvolvida por Pagani, Kovaleski e Resende (2015) com o auxílio dos softwares Zotero e JabRef-3.3. Esta pesquisa é caracterizada como quantitativa; do ponto de vista dos objetivos, classifica-se como exploratória; e quanto aos procedimentos técnicos, caracteriza-se como bibliográfica. Os resultados indicam que o maior número de publicações encontra-se na Sistemas de Suporte à Decisão (periódico Decision Support Systems, apresentando um percentual de 10\%). Conclui-se que os resultados desta pesquisa podem ser utilizados por pesquisadores que objetivem pesquisar sobre o assunto por proporcionar a otimização de tempo na busca pelas principais publicações e autores sobre o tema.

Palavras-chave: Processo decisório. Sistemas de informação. Contabilidade gerencial. Methodi Ordinatio.

\section{Introdução}

A controladoria é a área da organização cuja missão consiste em zelar pela eficácia do seu processo de gestão, tanto para finalidades internas como externas, desta forma, cuida para que os usuários disponham de todas as informações necessárias para que possam atingir plenamente os seus objetivos. Compreende a disseminação de conhecimento, modelagem e implantação de sistemas de informações, por meio da contabilidade gerencial e da contabilidade financeira. (FREZATTI; ROCHA; NASCIMENTO; JUNQUEIRA, 2009).

A gestão de uma empresa exige informações relevantes para o processo decisório, conforme Anthony e Govindarajan (2008). Entretanto, a partir da Era da Informação, com a disseminação da internet, têm-se observado os impactos da sobrecarga de informação no comportamento do indivíduo, no nível social e nos processos de tomada de decisão Gleick (2013); Santos (2011); Wurman (2005), que é o principal interesse deste estudo.

A expressão sobrecarga de informação é atribuída a Alvin Toffler, nos idos da década de 70. Para ele, superestimulação cognitiva interfere em nossa capacidade de pensar. (TOFFLER, 1971). Nesse sentido, Castells (1999) afirma que no seio da sociedade se opera uma revolução tecnológica concentrada nas tecnologias da informação que está remodelando sua base em ritmo acelerado. Ideia reforçada pela Lei de Moore que afirma que o processamento dos computadores tem sua capacidade dobrada a cada dezoito meses.

Dessa forma, para compensar situações de mudança rápida e irregular ou um

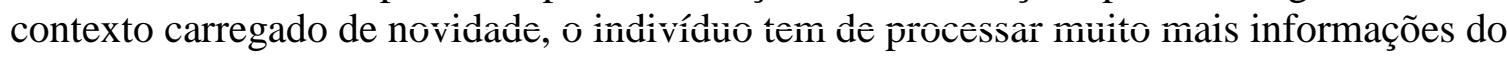
que antes para tomar decisões efetivas e racionais. (TOFFLER, 1971).

Atribui-se a Simon (1963), segundo Moritz (2006), o título de “pai” do Processo
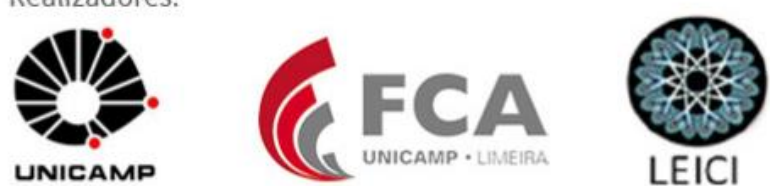
Decisório, cujos estudos propõem um modelo de decisão que admite a existência de uma racionalidade limitada. "O indivíduo coloca-se numa situação onde certos estímulos e certas classes de informações terão influência sobre ele”. (SIMON, 1970, p. 82).

Nesse sentido, o Modelo de Racionalidade Limitada (Bounded Rationality) adverte não ser possível para um tomador de decisão ter acesso a todas as possibilidades de ação devido à impossibilidade física de ter acesso a todas as informações e processá-las, além do alto custo envolvido nesse processo. (MORITZ, 2006).

Dito isto, este artigo visa responder a seguinte questão de pesquisa: Qual o estado do conhecimento sobre sobrecarga de informação na tomada de decisão em artigos científicos nos últimos dez anos?

Para responder a esta questão elaborou-se como objetivo: realizar um mapeamento de artigos científicos sobre sobrecarga de informação na tomada de decisão no período entre 2007 e 2016 com base na revisão de literatura do tipo "estado do conhecimento".

Justifica-se a relevância deste estudo considerando que, conforme o Estágio 2 do pronunciamento International Management Accounting Practice 1 (IMAP 1), emitido em 1998, pela International Federation of Accountants - IFA, observa-se um destaque definitivo no suprimento de informações através de tecnologias, ênfase na análise do processo decisório e contabilidade por responsabilidade. (INTERNATIONAL FEDERATION OF ACCOUNTANTS, 1998). Ainda, espera-se com esta pesquisa colaborar com o conhecimento científico, dado que o incipiente número de publicações está em proporção inversa à intensificação do tema proporcionada pelos atuais desenvolvimentos da Tecnologia da Informação e Comunicação (TIC) e seus efeitos sobre os indivíduos e a sociedade, bem como apresentar um portfólio de artigos a fim de divulgar um estudo inicial sobre o tema sobrecarga de informação.

O artigo está estruturado da seguinte forma, na próxima seção é apresentado o referencial teórico, que dará sustentação à pesquisa, a seção 3 descreve a metodologia utilizada, na seção 4 são demonstrados e discutidos os resultados encontrados e por fim, a seção 5 com as considerações finais e sugestões de estudos futuros.

\section{Referencial Teórico}

Esta seção apresenta os conceitos fundamentais norteadores do estudo cuja intenção é fornecer os subsídios mínimos para a compreensão da pesquisa e seu contexto.

\subsection{Processo de Tomada de Decisão}

De acordo com Mintzberg, Raisinghani e Théorêt (1976), a tomada de decisão requer a implementação de uimi processo decisónió, forimaal ou infớminal, estruturado ou não estruturado que se inicia com uma ação e termina com uma decisão.

Neste sentido, Barros e Herbert (2010) argumentam que "o conceito de racionalidade processual é o que melhor expressa a visão de Simon do comportamento racional, em detrimento da racionalidade limitada [...]" (BARROS E HERBERT, 2010, p. 456). Em suma, eles destacam que os conceitos de racionalidade limitada (bounded rationality), defendido por Herbert Simon desde o início de sua carreira, e racionalidade processual são usados por Simon como complementares. 
Na perspectiva atual, Butler et al. (2015) busca sistematizar as várias visões sobre como a neurociência cognitiva organizacional pode contribuir para aprofundar a compreensão do processo de tomada de decisão gerencial, por meio de uma ampla revisão de literatura recente e oferece reflexões sobre as perspectivas futuras sobre a neurociência cognitiva afirmando que "com esse conhecimento podemos compensar os limites de nossa tomada de decisões e melhorar a nossa autodeterminação para influenciar a forma como trabalhamos". (BUTLER et al., 2015, p.16).

\subsection{Da Informação à Sobrecarga de Informação}

A informação também foi objeto de estudo da Ciência Cognitiva, embora seja polêmica por ser acusada de apresentar uma visão mecanicista da mente, ela tem contribuído, de forma significativa, na atualidade, para a geração de artefatos informacionais, em especial na Robótica e na Inteligência Artificial.

Nessa perspectiva, a concepção de informação desenvolvida por Shannon e Weaver (1964), cuja preocupação central é com a quantificação da informação, visa estabelecer a quantidade de informação em uma fonte, a fim de que ela possa ser transmitida do modo mais eficiente possível. Dessa forma, afirmam que quanto mais desordenado for um sistema, maior será o seu grau de entropia ${ }^{1}$.

Para pensadores como Le Coadic (1996), um modelo do processo de comunicação humana deve ser circular, sem a presença da fonte e destino. Todos os participantes do processo comunicativo informam e são informados ao mesmo tempo. Assim, todos possuem o mesmo poder de escolha e transmissão da mensagem. A informação é construída, usada e comunicada por todos simultaneamente, gerando, em princípio, ações coletivas e responsáveis. Nesse modelo, os participantes do processo comunicativo possuem acesso democrático ao conjunto de mensagens possíveis ou disponíveis, podem, idealmente, utilizá- las de modo igualitário e comunicá-las em benefício da coletividade.

O termo sobrecarga de informação é definido por Bawden, Holtham e Courtney (1999) da seguinte forma:

Não há uma única definição de sobrecarga de informação aceita de forma generalizada. O termo é usualmente tido como descritivo de um estado de coisas onde a eficiência do indivíduo em usar a informação em seu trabalho é perturbada pela quantidade de informação relevante e potencialmente útil disponível. [...] O conceito é associado com alguma perda de controle sobre a situação e com sensações de desnorteamento. No limite, pode levar a problemas de saúde. (BAWDEN ; HOLTHAM; COURTNEY, 1999, p. 249).

Nesse sentido, Wurman (2005) afirma que "o exagero na quantidade de informação começa a nublar as diferenças entre dados e informação, entre fatos e conhecimentos" (WURMAN, 2005, p. 17). O autor continua, "fazendo com que nossos canais de percepção entrem em curto-circuito" (WURMAN, 2005, p. 17).

Contudo, há um grupo de pensadores que além de pensarem a sobrecarga de informação como benéfica, contradizem a ideia de que ela seja um fenômeno contemporâneo conforme afirma Rosenberg (2003), no Journal of the History of Ideas, que reúne uma série de antigós soburic o temina:

${ }^{1}$ Para a Teoria da Informação a entropia é interpretada como uma medida do grau de incerteza que existe antes que uma escolha seja feita. (BRILLOUIN, 1962).

\section{Organizadores:}

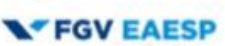

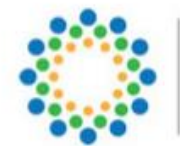

ANEGEPE

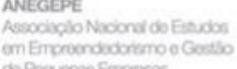


A noção de sobrecarga de informação aparece em todo lugar na mídia popular como uma caracterização de algo específico e emblemático da nossa era, da vida em um tempo de celulares e navegadores de internet e aparelhos de fax e inumeráveis outros 'aparelhos de informação'. (ROSENBERG, 2003, p. 1).

Este grupo associa as pesquisas sobre a sobrecarga de informação à Ciência da Informação e corrobora com os achados de Neil (1992) que afirmaram que "[...] para bibliotecários e cientistas da informação, a sobrecarga de informação era uma oportunidade, um desafio, uma chance de fazer o que fazem melhor". (NEIL, 1992, p. 100).

\subsection{Estado do Conhecimento}

Diante do atual cenário de excesso e rapidez de informações, Coelho e Silva (2013) destacam que as revisões de literatura, tornam-se muito mais eficazes para conhecimento de um objeto e evitam o desperdício de tempo que se consome na tentativa de identificar se determinado estudo já foi realizado anteriormente, podendo, assim, o pesquisador se dedicar especificamente ao objeto de estudo de fato.

Os trabalhos de revisão são definidos como investigações que analisam a produção bibliográfica de determinada área temática, "dentro de um recorte de tempo, que fornece uma visão geral ou um relatório de "estado da arte" sobre um tópico específico, evidenciando novas ideias, métodos, subtemas, marcos temporais". (NORONHA; FERREIRA, 2000, p. 191).

Nesse cenário, a produção brasileira de pesquisa do tipo "estado da arte" fortaleceuse, como relatam Fávero e Oliveira (2012), por iniciativa e com apoio financeiro do Inep. Em colaboração com os centros de pesquisa da área, sobretudo, com grupos de trabalho da Anped, que se encarregaram da elaboração da maior parte dos números, foram publicados onze números na série Estado do Conhecimento.

A exemplo de diversos casos da literatura, esta pesquisa considera os termos "estado da arte" e "estado do conhecimento" como sinônimos. Assim, utiliza-se das citações de vários autores e menciona estes termos de forma indiscriminada.

Na próxima seção serão apresentados os procedimentos metodológicos utilizados para elaboração da revisão da relação entre estes temas nos estudos atuais.

\section{Metodologia}

Esta pesquisa propôs descrever o estado do conhecimento sobre sobrecarga de informação na tomada de decisão em artigos científicos no período entre 2007 e 2016. Com relação à sua natureza, classifica-se como básica; quanto à abordagem do problema, classifica-se como quantitativa; do ponto de vista dos objetivos, classifica-se como exploratória; e quanto aos procedimentos técnicos, classifica-se como bibliográfica. (SILVA; MENEZES, 2005).

Os dados analisados foram obtidos de fontes primárias, são elas: bases de dados ScienceDirect e EBSCOHost. Os dados foram coletados em dezembro de 2017 a partir da busca por artigos de periódicos nos idiomas inglês e português que contivessem os termos 'making-decision' e 'information overload' no título dos trabalhos, no resumo e nas palavras- chave em todos os anos. A seleção ocorreu pelo uso dessas palavras mais o conectivo booleano $\mathrm{E}(A N D)$ para a formulação da expressão booleana. Na pesquisa definitiva foram aplicados filtros diretamente nas bases para a seleção de resultados apenas de trabalhos nas áreas de business, management accounting e social sciences. Neste
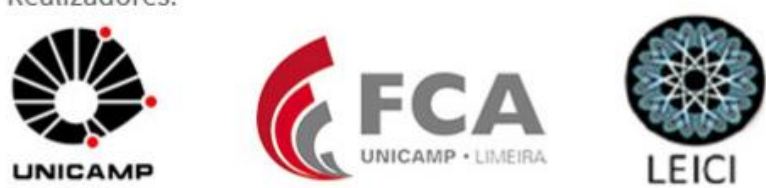
sentido, as bases trouxeram 929 artigos. Na sequência, fez-se a migração dos artigos para o software de gestão de referências Zotero para a retirada dos artigos duplicados. Assim sendo, os artigos totalizaram 553 (deste número, 29 foram eliminados por apresentarem informações incompletas). Em seguida, através da leitura de cada título, identificou-se os artigos cujos títulos não estavam alinhados ao tema pesquisado e eles foram retirados. Após a filtragem ficaram 28 artigos para classificação através da técnica Methodi Ordinatio de Pagani, Kovaleski e Resende (2015). Na fase seguinte, buscou-se identificar o fator de impacto, ano e número de citações, os dados dos artigos arquivados no Zotero foram importados para o Excel através do gerenciador de referências JabRef. As fontes utilizadas para essa atividade foram o Google Scholar e os sites dos periódicos. Para todos os fatores de impacto utilizou-se a métrica JCR do último ano, 2016, portanto. Ressalta-se que, para os fatores de impacto não localizados, atribuiu-se o valor zero, conforme orientação da coautora, Regina N. Pagani, por contato através da página https://www.facebook.com/methodiordinatio/. Por fim, foi aplicada a equação InOrdinatio. Para a seleção do protfólio, foi atribuído o valor 10, buscando identificar e valorizar publicações recentes sobre o tema.

Segundo Soares, Kovaleski e Pagani (2017), a equação InOrdinatio leva em consideração o fator de impacto da revista de publicação do artigo, o ano da publicação do artigo e a quantidade de citações do documento e é expressa da seguinte forma:

$$
\text { InOrdinatio }=(\mathrm{Fi} / 1000)+\alpha^{*}[10-(\text { AnoPesq }- \text { AnoPub })]+\left(\sum \mathrm{Ci}\right)
$$

Na qual, Fi é o fator de impacto, $\alpha$ é um fator de ponderação variando de 1 a 10, a ser atribuído pelo pesquisador; AnoPesq é o ano em que a pesquisa foi desenvolvida; AnoPub é o ano em que o artigo foi publicado; e $\sum$ Ci é o número de vezes que o documento foi citado.

Finalmente, há que se esclarecer que as bases foram escolhidas por conveniência, por estarem à disposição da instituição a qual os autores da presente pesquisa possuem vínculo. Estabeleceu-se um recorte temporal referente aos anos de 2007 a 2016. Buscouse a técnica Methodi Ordinatio devido ao fato de que, diferente de outros métodos disponíveis, neste, o pesquisador tem critérios para classificar a relevância do artigo encontrado ao ranquear os artigos essenciais através dos critérios: fator de impacto, número de citação e ano de publicação. Considerando-se o objetivo deste estudo as duas últimas fases da metodologia escolhida não foram aplicadas.

A Tabela 1 mostra o resultado da pesquisa definitiva nas bases de dados. 


\section{TABELA 1 - RESULTADOS BRUTOS DA BUSCA SISTEMÁTICA (ANTES DA} FILTRAGEM).

\begin{tabular}{|c|c|c|c|}
\hline \multirow{2}{*}{$\begin{array}{c}\text { PALAVRAS } \\
\text { CHAVE }\end{array}$} & \multicolumn{2}{|c|}{ BASE DE DADOS } & \multirow[b]{2}{*}{ TOTAL } \\
\hline & SCIENCEDIRECT & EBSCOHost & \\
\hline $\begin{array}{l}\text { Making- } \\
\text { decision } \\
\text { Information } \\
\text { overload }\end{array}$ & $\begin{array}{c}\text { Localizar todos meus } \\
\text { termos: (decision-making) } \\
\text { AND (information overload) } \\
\text { Limitadores: Data de } \\
\text { publicação: } \\
\text { 20070101- } \\
\text { 20161231 Disponível na } \\
\text { Library Collection Idioma: } \\
\text { English, Portuguese Tipos de } \\
\text { Fontes: Revistas } \\
\text { acadêmicas. }\end{array}$ & $\begin{array}{c}\text { Search results: } 280 \\
\text { results found for } \\
\text { (docsubtype(FLA) and pub-date } \\
>2007 \text { and pub-date }<2016 \text { and } \\
\text { ALL(decision-making) and } \\
\text { ALL(information overload)) and } \\
\text { not (itemstage(S5) or } \\
\text { itemstage(S100) or } \\
\text { itemstage(S200)) AND LIMIT- } \\
\text { TO(topics, "organization,social”). }\end{array}$ & 929 \\
\hline Total & 649 & 280 & \\
\hline
\end{tabular}

FONTE: Dados da pesquisa (2017).

Para a geração do portfólio sobre o tema de interesse da pesquisa foi aplicada a técnica Methodi Ordinatio. Pagani, Kovaleski e Resende (2015) desenvolveram esta metodologia que afirma que quanto maior for o valor de InOrdinatio, mais relevante será o artigo para o portfólio.

A Figura 1 ilustra o procedimento desta metodologia de forma resumida. 
FIGURA 1 - PROCEDIMENTO METHODI ORDINATIO RESUMIDO PARA OBTENÇÃO DO PORTFÓLIO BIBLIOGRÁFICO

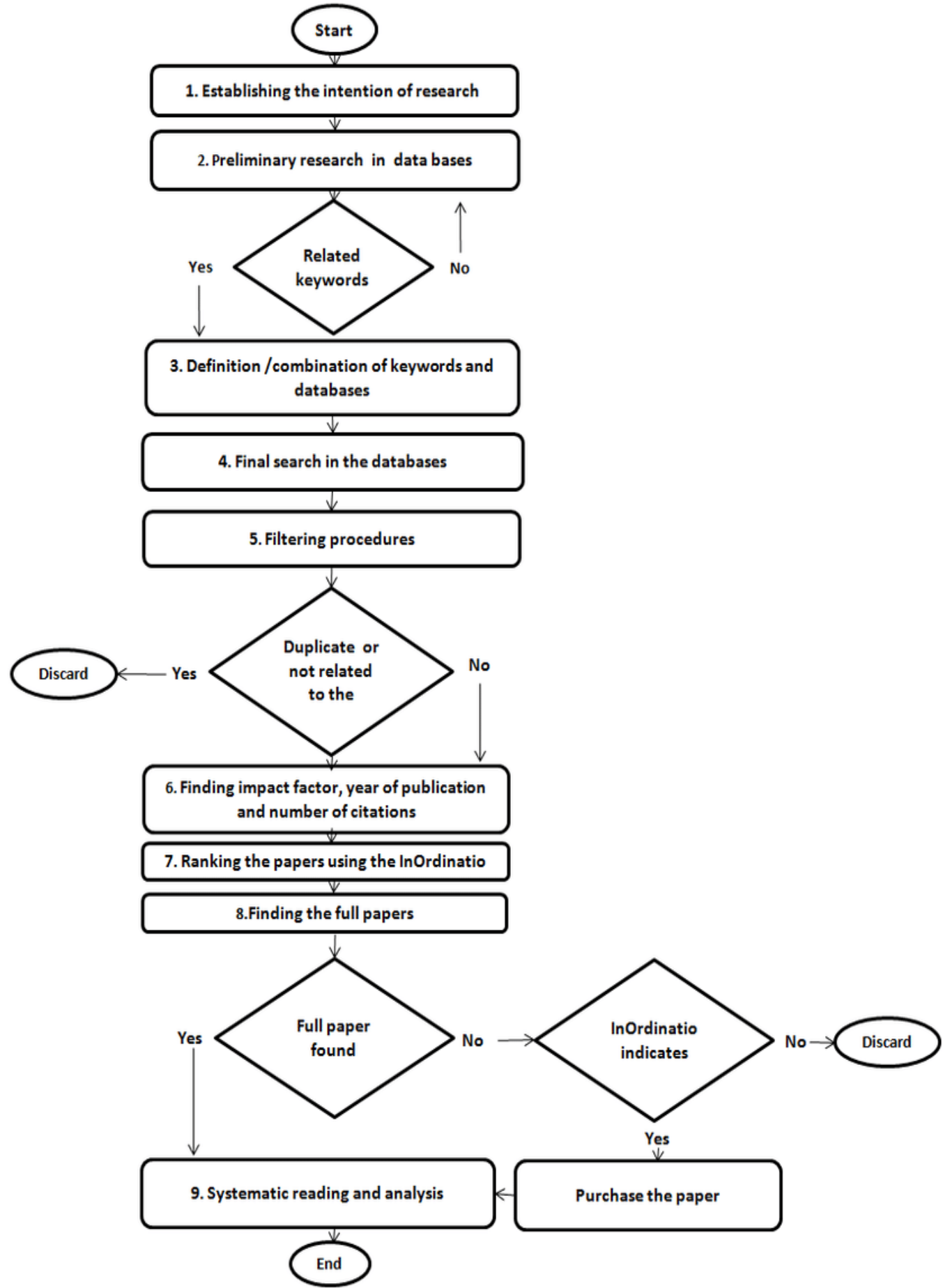

FONTE: PAGANI, KOVALESKI E RESENDE (2015). 
Como pode ser visto na Figura 1, a metodologia é composta por 9 fases, são elas: Fase 1 - Estabelecimento da intenção de pesquisa; Fase 2 - Pesquisa preliminar de palavras-chave em bases de dados; Fase 3 - Definição e combinação de palavras-chave e bases de dados; Fase 4 - Pesquisa definitiva nas bases de dados; Fase 5 - Procedimentos de filtragem; Fase 6 Identificação do fator de impacto, ano e número de citações; Fase 7 - Ordenação dos artigos por meio da equação InOrdinatio; Fase 8 - Localizar os artigos em formato integral e Fase 9 Leitura Sistemática dos artigos.

\section{Análise dos Dados e Discussão dos Resultados}

A apresentação do gráfico possibilita a visualização da quantidade de artigos, com os termos 'making-decision' e 'information overload', distribuídos ao longo dos anos, estes resultados são ilustrados na Figura 2.

\section{FIGURA 2 - PUBLICAÇÕES POR ANO}

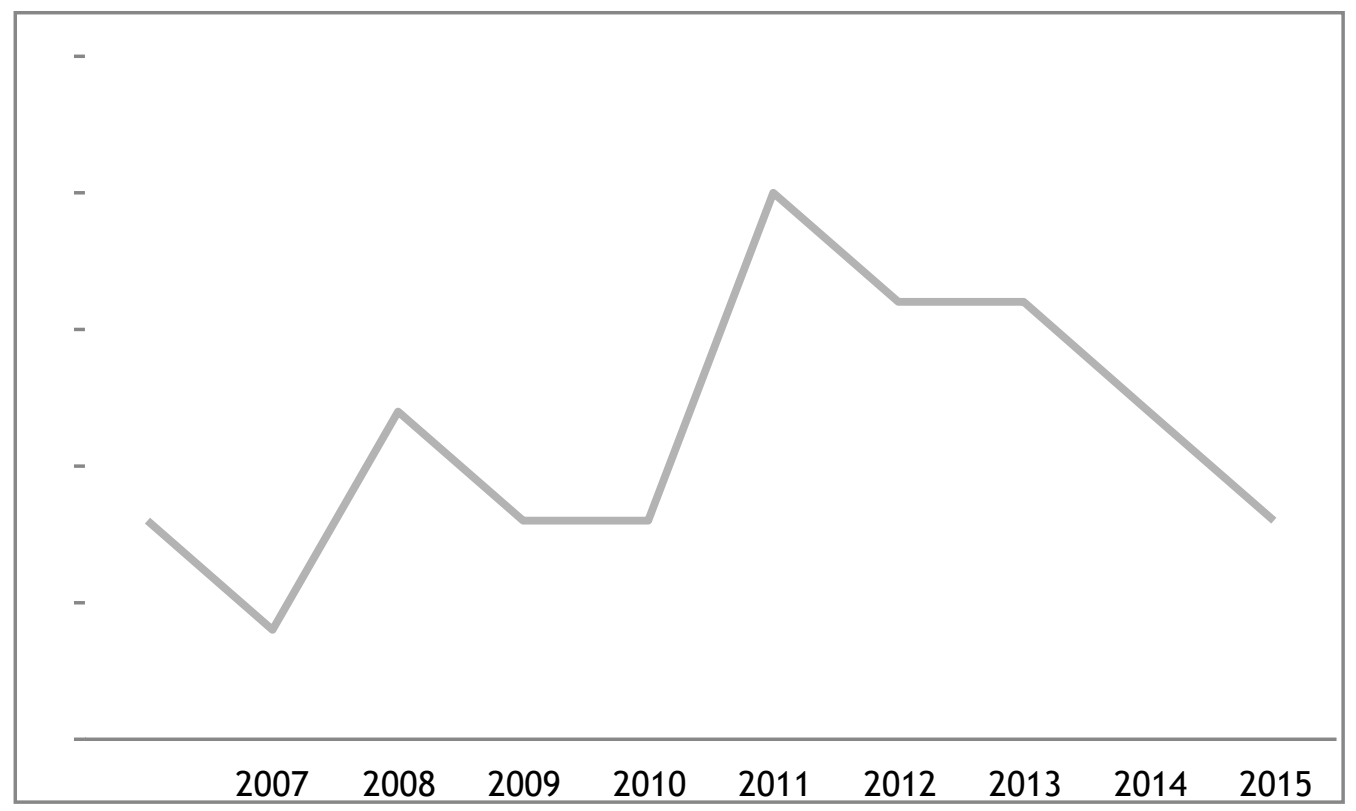

FONTE: Os autores (2018).

Nota-se que, as publicações encontradas nas bases mostram crescimento neste período, apesar da presença de ciclos de baixa a cada ano com pico em 2012. Vale ressaltar que o ano de 2017 foi excluído da comparação porque o número de artigos seria artificialmente baixo, devido ao fato de que a busca foi realizada ainda no decurso deste ano. Constata-se que ainda há um baixo número de publicações sobre o tema na atualidade.

A Figura 3 apresenta as principais fontes de publicação sobre o tema. 


\section{FIGURA 3 - FONTES DE PUBLICAÇÃO}

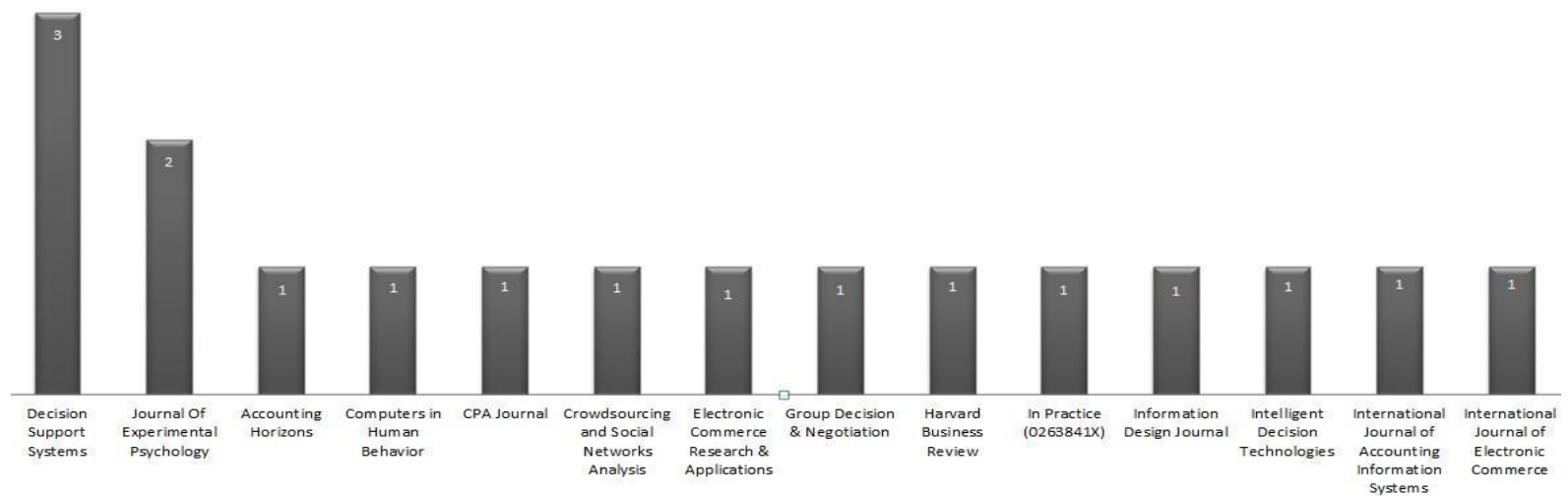

FONTE: Os autores (2018).

Observa-se que o periódico Decision Support Systems possui o maior número de publicações, 3 artigos (10\%), seguido do Journal Experimental Psychology, 2 artigos $(6,57 \%)$ e os demais, 1 artigo $(3,09 \%)$ cada.

A Figura 4 mostra as publicações por autores mais citadas no Google Acadêmico.

\section{FIGURA 4 - CITAÇÕES GOOGLE ACADÊMICO}

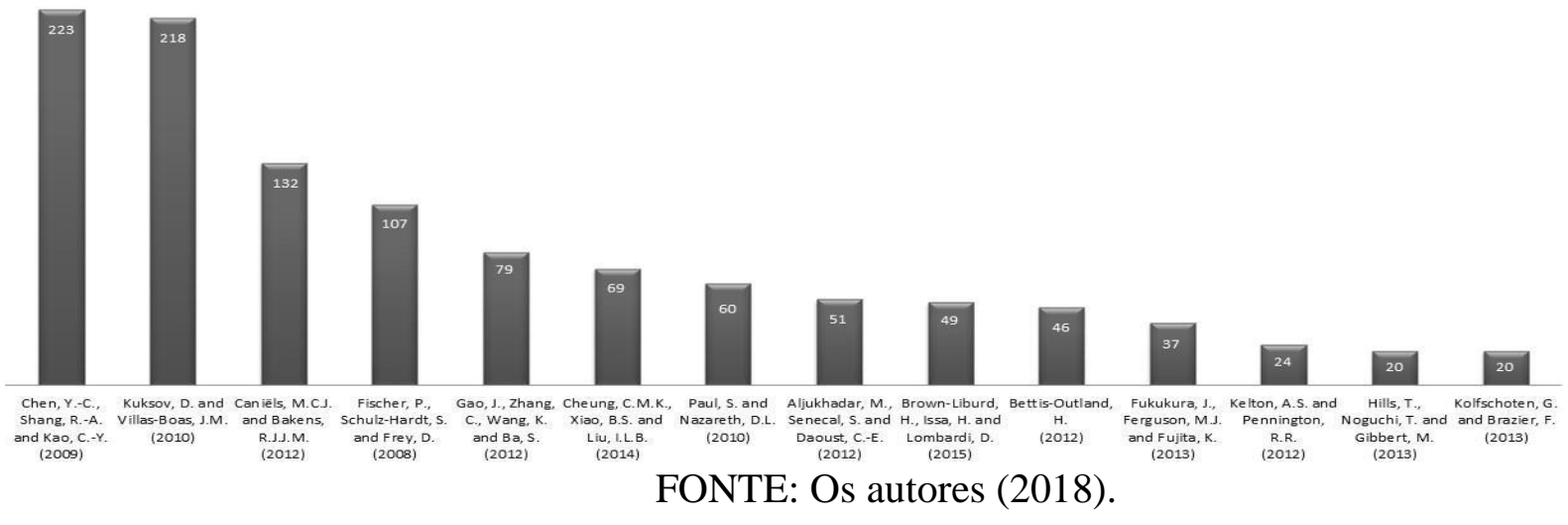

Chen, Y. C., Shang, R. A. e Kap, C. Y. (2009) apresentam uma quantidade destacada de citações. As publicações dos autores Kuksov, D. e Villas-Boas, J. M. (2010); Caniëls, $\mathrm{M}$.

C. J. e Bakens, R. J. J. M. (2012); Fischer, P., Schulz-Hardt, S. e Frey, D. (2008) e Gao, J. Zhang, C., Wag, k. e Ba, S. (2012) também demonstram citações elevadas no site consultado.

A Tabela 2 evidencia os principais artigos sobre tomada de decisão e sobrecarga de informação resultantes das fases 1 a 7 da metodologia Methodi Ordinatio. 
TABELA 2 - PORTFÓLIO DE ARTIGOS SOBRE MAKING-DECISION E INFORMATION OVERLOAD.

\begin{tabular}{|c|c|c|c|c|c|}
\hline Classificação & $\begin{array}{c}\text { Artigos sobre making-decision e } \\
\text { information overload (autores, ano, } \\
\text { periódico) }\end{array}$ & $\begin{array}{l}\text { Fator de } \\
\text { Impacto }\end{array}$ & Citações & Ano & InOrdin \\
\hline 1 & $\begin{array}{c}\text { Kuksov, D. and Villas-Boas, J.M. (2010). When } \\
\text { More Alternatives Lead to Less Choice. } \\
\text { Marketing Science }\end{array}$ & 2,163 & 218 & 2010 & 258,00 \\
\hline 2 & $\begin{array}{l}\text { Chen, Y.-C., Shang, R.-A. and Kao, C.-Y. } \\
\text { (2009). The effects of information overload } \\
\text { on consumers' subjective state towards } \\
\text { buying decision in the internet shopping. } \\
\text { Electronic Commerce Research \& } \\
\text { Applications environment. }\end{array}$ & 0,000 & 223 & 2009 & 253,00 \\
\hline 3 & $\begin{array}{l}\text { Caniëls, M.C.J. and Bakens, R.J.J.M. } \\
\text { (2012). The effects of Project } \\
\text { Management Information Systems on } \\
\text { decision making in a multi project } \\
\text { environment. International Journal of } \\
\text { Project Management. }\end{array}$ & 0,000 & 132 & 2012 & 192,00 \\
\hline 4 & $\begin{array}{l}\text { Cheung, C.M.K., Xiao, B.S. and Liu, } \\
\text { I.L.B. (2014). Do actions speak louder than } \\
\text { voices? The signaling role of social } \\
\text { information cues in influencing consumer } \\
\text { purchase decisions. Crowdsourcing and } \\
\text { Social Networks Analysis. }\end{array}$ & 0,000 & 69 & 2014 & 149,00 \\
\hline \multirow[t]{2}{*}{5} & $\begin{array}{l}\text { Gao, J., Zhang, C., Wang, K. and Ba, S. (2012). } \\
\text { Understanding online purchase decision } \\
\text { making: The effects of unconscious thought, } \\
\text { information quality, and information } \\
\text { quantity. Decision Support Systems. }\end{array}$ & & & & \\
\hline & & 3,222 & 79 & 2012 & 139,00 \\
\hline 6 & $\begin{array}{l}\text { Brown-Liburd, H., Issa, H. and Lombardi, } \\
\text { D. (2015). Behavioral Implications of Big } \\
\text { Data's Impact on Audit Judgment and } \\
\text { Decision Making and Future Research } \\
\text { Directions. Accounting Horizons. }\end{array}$ & 1,218 & 49 & 2015 & 139,00 \\
\hline 7 & $\begin{array}{l}\text { Fischer, P., Schulz-Hardt, S. and Frey, D. } \\
\text { (2008). Selective Exposure and Information } \\
\text { Quantity: How Different Information } \\
\text { Quantities Moderate Decision Makers' } \\
\text { Preference for }\end{array}$ & 0,000 & 107 & 2008 & 127,00 \\
\hline
\end{tabular}

Artigos sobre making-decision e periódico)
Fator de

Impacto Citações Ano InOrdin 
Consistent and Inconsistent Information. Journal of Personality \& Social Psychology.

Aljukhadar, M., Senecal, S. and Daoust, C.-E. (2012). Using Recommendation Agents to Cope with Information Overload. International Journal of Electronic Commerce.

Fukukura, J., Ferguson, M.J. and Fujita, K. (2013). Psychological distance can improve decision making under information overload via gist memory. Journal of Experimental Psychology: General.

Moges, H.-T., Vlasselaer, V.V., Lemahieu, W. and Baesens, B. (2016). Determining the use of data quality metadata (DQM) for decision making purposes and its impact on decision outcomes -- An exploratory study.

Decision Support Systems.

$\begin{array}{llll}3,222 & 7 & 2016 & 107,00\end{array}$

11 Murayama, K., Blake, A.B., Kerr, T. and

Castel, A.D. (2016). When enough is not enough: Information overload and metacognitive decisions to stop studying information. Journal of Experimental Psychology. Learning, Memory, And Cognition.

Bettis-Outland, H. (2012). Decisionmaking's impact on organizational learning and information overload. Journal of Business Research.

$\begin{array}{llll}3,354 & 46 & 2012 & 106,00\end{array}$

Paul, S. and Nazareth, D.L. (2010). Input information complexity, perceived time pressure, and information processing in GSS-based work groups: An experimental investigation using a decision schema to alleviate information overload

$$
\text { conditions. }
$$

Decision Support Systems. 
Rosen, L. and Samuel, A. (2015).

Conquering digital distraction: two experts on managing the overload.

$$
\text { Harvard Business Review. }
$$

7

2015

97,00

15

Goswami, S. (2015). Analysing Effects of Information Overload on Decision Quality in an Online Environment. Journal of Management Research. 0,000 5 2015 95,00

16

Hadar, L. and Sood, S. (2014). When Knowledge Is Demotivating: Subjective

Knowledge and Choice Overload. Psychological Science.

Hills, T., Noguchi, T. and Gibbert, M. (2013). Information overload or search-amplified risk? Set size and order effects on decisions from experience.

Psychonomic Bulletin \& Review.

Kolfschoten, G. and Brazier, F. (2013).

Cognitive Load in Collaboration:

Convergence. Group Decision \&

Negotiation.

0,000

20

2013

19 Kelton, A.S. and Pennington, R.R. (2012). Internet financial reporting: The effects of information presentation format and content differences on investor decision making. Studia Psychologica.

BALÁŽ, V., BAČOVÁ, V. and ŠKRINIAR, P. (2014). EXPERIENCE AND INFORMATION SEARCH PATTERNS IN COMPLEX DECISION MAKING. Computers in Human Behavior.

\section{0,511}

2

2014

82,00

21 Ito, G., Shibata, T. and Takano, Y. (2014). The Use of a Foreign Language Can Improve Decision Making Under Information Overload. Proceedings of the Cognitive Science Society. 
Technologies.

\begin{tabular}{|c|c|c|c|c|c|}
\hline 23 & $\begin{array}{l}\text { Wiltermuth, S.S. and Neale, M.A. (2011). } \\
\text { Too Much Information: The Perils of } \\
\text { Nondiagnostic Information in } \\
\text { Negotiations. Journal of Applied } \\
\text { Psychology. }\end{array}$ & 4,130 & 18 & 2011 & 68,00 \\
\hline 24 & $\begin{array}{l}\text { Green, A. (2011). How the Information } \\
\text { overload in healthcare management: } \\
\text { READ Portal is helping healthcare } \\
\text { managers. Journal of the Canadian } \\
\text { Health Libraries Association (JCHLA). }\end{array}$ & 0,000 & 4 & 2011 & 54,00 \\
\hline 25 & $\begin{array}{l}\text { Wheeler, P. and Arunachalam, V. } \\
\text { (2009).The effects of multimedia on } \\
\text { cognitive aspects of decision-making. } \\
\text { International Journal of Accounting } \\
\text { Information Systems. }\end{array}$ & 0,000 & 18 & 2009 & 48,00 \\
\hline 26 & $\begin{array}{l}\text { Jenkins, J. (2009). From data and } \\
\text { measures to meaningful decisions: } \\
\text { Designing useful information for senior } \\
\text { managers and boards. Information } \\
\text { Design Journal. }\end{array}$ & 0,000 & 5 & 2009 & 35,00 \\
\hline 27 & $\begin{array}{l}\text { Epstein, B.J. (2007). Information } \\
\text { Overload Can Threaten Sound Decision- } \\
\text { Making. CPA Journal. }\end{array}$ & 0,000 & 18 & 2007 & 28,00 \\
\hline 28 & $\begin{array}{l}\text { Girotti, R. (2007). Information overload? } \\
\text { Tools for tactical decision making. In } \\
\text { Practice. }\end{array}$ & 0,050 & 0 & 2007 & 10,00 \\
\hline
\end{tabular}

FONTE: Os autores (2018).

Do conjunto geral de dados obtidos foram elencados os artigos essenciais pelos critérios (fator de impacto, número de citações e ano de publicação).

Esta seção apresentou discussão sobre os resultados do presente estudo obtidos por meio da técnica Methodi Ordinátio. Na próxima seção, serão apresentadas as considerações finais, as limitações do estudo e as sugestões de estudos futuros.

\section{Considerações Finais, Limitações do Estudo e Pesquisas Futuras}

O presente artigo teve como objetivo geral realizar um mapeamento de artigos científicos sobre sobrecarga de informação na tomada de decisão no período entre 2007 e 2016 com base na revisão de literatura do tipo "estado do conhecimento". Para atingir 
este geral foram delineados quatro objetivos específicos. Os achados obtidos através dos objetivos específicos são apresentados no parágrafo a seguir.

O primeiro objetivo específico foi identificar a quantidade de artigos pertinentes à sobrecarga de informação na tomada de decisão nas bases de dados científicos. Os resultados do estudo revelam um baixo número de publicações, fato que se encontra em proporção inversa aos atuais desenvolvimentos da Tecnologia da Informação e Comunicação (TIC) cujos efeitos são facilmente identificados tanto no nível da sociedade quanto no nível do indivíduo. Efeitos que, por si só, podem indicar consistentes demandas por novos estudos.

O segundo objetivo específico teve como foco apresentar as principais fontes de publicação sobre o tema. A partir dos resultados deste estudo é possível afirmar que o maior número de publicações encontra-se na área de Sistemas de Suporte à Decisão (periódico Decision Support Systems, apresentando um percentual de 10\%). Este achado aproxima-se da ideia de controle gerencial de Anthony e Govindarajan (2008) enquanto um conjunto de atividades suportadas pela Contabilidade Gerencial através de um sistema de informações. O segundo maior número de publicações encontra-se na área da Psicologia (Journal Experimental Psychology, 6,57\%), área congênere da Contabilidade, sobretudo, nos estudos de contabilidade comportamental.

O terceiro objetivo específico teve como proposta mostrar as publicações por autores mais citados no Google Acadêmico. A partir dos resultados do estudo pode-se conhecer os autores que possuem maior visibilidade e difusão na área. $\mathrm{O}$ número de citações é um índice que possui um alto nível de confiança pela comunidade acadêmica global, ao mesmo tempo que é controverso se for utilizado como métrica de qualidade da pesquisa.

Gerar um portfólio de artigos sobre o tema foi o propósito do quarto e último objetivo. A tabela associada a esse objetivo mostra os artigos mais relevantes para uma leitura e uma análise sistemática do tema de pesquisa. Asssim, este resultado está em consonância com os achados de Soares e Maciel (2000) que atestam que uma metodologia adequada para o levantamento e avaliação do conhecimento de qualquer fenômeno deve prever um inventário da produção acadêmica e científica onde se identifique as diversas perspectivas com as quais o estudo do tema tem sido enriquecido ao longo do tempo.

Conclui-se, a partir das discussões anteriores, que o estado do conhecimento sobre sobrecarga de informação na tomada de decisão, devido ao ainda incipiente número de publicações sobre o tema, demanda a realização de novos estudos com a atualização de dados e sua validação pelos pesquisadores e pela academia.

Uma das limitações deste estudo diz respeito ao fato de apenas apresentar o interesse de várias áreas pelo tema, no entanto, sem aprofundá-lo. Desta forma, pesquisas futuras podem ser direcionadas a analisar as contradições e, sobretudo, as lacunas, isto é, os aspectos não estudados ou ainda estudados de forma precária, bem como a identificação de metodologias de pesquisa pouco utilizadas.

Devido ao fato do estudo não ser estatístico, uma vez que apenas se utilizou de uma técnica estatística, os resultados não podem ser generalizados, devem, portanto, ser tomados apenas como indicativos. Com isto, futuros estudos podem realizar as devidas mensurações estatísticas visando sua generalização. Não obstante, podem avançar para estudos de
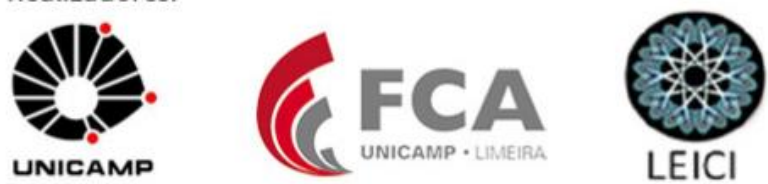
Análise de Redes Sociais (ARS) na produção científica através dos quais é possível investigar sobre as redes sociais e as suas redes de cooperação, evidenciando, assim, a evolução da produção científica na referida área do conhecimento através de laços relacionais.

\section{REFERÊNCIAS}

ANTHONY, R. N.; GOVINDARAJAN, V. Sistemas de controle gerencial. 12. ed. São Paulo: McGraw-Hill. 2008.

BARROS, G.; HERBERT, A. Simon and the concept of rationality: boundaries and procedures. Brazilian Journal of Political Economy, v. 30, p. 455-472, Jul./Sep. 2010.

BAWDEN, D.; HOLTHAM, C.; COURTNEY, N. Perspectives on information. Aslib Proccedings, v. 51. n.8, p. 249-255, 1999.

BRILLOUIN, L. Science and Information Theory, $2^{\text {nd }}$. Ed. New York: Academic Press, 1962.

BUTLER, M. J. et al. How organizational cognitive neuroscience can deepen understanding of managerial decision-making: a review of the recent literature and future directions. International Journal of Management Reviews, v.0, p. 1-18. 2015.

CASTELLS, M. Sociedade em rede. São Paulo: Paz e terra. 1999.

COELHO, W. N.; SILVA, R. M. Relações raciais e educação: o estado da arte. Revista Teias, v.14, p. 121-146. 2013.

FÁVERO, O.; OLIVEIRA, R. A. Estado da arte e disseminação da pesquisa educacional: nota dos organizadores. Em aberto, v.25, p. 189-191, jan./jun. 2012.

FREITAS, G. L. A sociedade da informação no discurso dos pesquisadores em educação: análise de conteúdo de periódicos. RBBD. Revista Brasileira de Biblioteconomia e Documentação, v. 13, p. 2350-2373, 2017.

GLEICK, J. A informação: uma história, uma teoria, uma enxurrada. São Paulo: Companhia das Letras. 2013.

INTERNATIONAL FEDERATION OF ACCOUNTANTS. International Management Accounting Practice 1 (IMAP 1). 1998.

LE COADIC, Y. F. A Ciência da Informação. Tradução de Maria Yeda F. S. de Figueiredo Gomes. Brasília, DF: Briquet de Lemos. 1996. 
MINTZBERG, H.; RAISINGHANI, D.; THÉORÊT, A. The structure of unstructured decision processes. Administrative Science Quarterly, v.21, pp. 246-275. 1976.

MORITZ, G. O. Processo decisório. Florianópolis: SEAD/UFSC. 2006.

NEILL, S. D. The dilema of information overload: managing in the Information Society. In: . Dilemmas in the study of Information: exploring the boundaries of Information Science. Westport: Greenwood Press, p. 99-158. 1992.

NORONHA, D.; FERREIRA, S. Revisões de literatura. In: B. Campello, B. Condon, \& J. Kremer, Fontes de informação para pesquisadores e profissionais. Belo Horizonte: UFMG. 2000.

PAGANI, R. N.; KOVALESKI, J. L.; RESENDE, L. M. Methodi Ordinatio: a proposed methodology to select and rank relevant scientific papers encompassing the impact factor, number of citation, and year of publication. Scientrometrics, v. 105, n.3, p. 2109-2135. 2015.

ROSENBERG, D. Early modern information overload. Journal of the History of Ideas, v. 64, n. 1, p. 1-9, jan. 2003.

SANTOS, L. G. Politizar as novas tecnologias: o impacto sociotécnico da informação digital e genética. São Paulo: Editora 34. 2011.

SHANNON, C. E.; WEAVER, W. The mathematical Theory of Communication. The University of Illinois Press. Urbana, 1964.

SILVA, E. L.; MENEZES, E. M. Metodologia da pesquisa e elaboração de dissertação. 4. ed. Florianópolis: UFSC. 2005.

SIMON, H. A. A capacidade de decisão e liderança. Rio de Janeiro: Fundo de Cultura. 1963.

SIMON, H. A. Comportamento administrativo. Estudo dos processos decisórios nas organizações. 2. ed. Rio de Janeiro: Fundação Getúlio Vargas. 1970.

SOARES, A. J.; KOVALESKI, J. L.;PAGANI, R. N. A geração de um portfólio de artigos sobre o tema melhoria contínua através da metodologia Methodi Ordinatio. Anais do VII Congresso Brasileiro de Engenharia de Produção. Dez. 2017. 
SOARES, M. B.; MACIEL, F. Alfabetização. Série Estado do Conhecimento. Brasília: MEC/Inep/Comped. 2000.

TOFFLER, A. Future shock. New York: Bantam. 1971.

WURMAN, R. S. Ansiedade de informação 2. São Paulo: Editora Cultura. 2005. 